\title{
Optimal timing of biodiversity offsetting for metapopulations
}

\author{
Darren M. Southwell, ${ }^{1,3}$ Geoffrey W. Heard, ${ }^{2}$ and Michael A. McCarthy ${ }^{1}$ \\ ${ }^{1}$ Australian Research Council Centre of Excellence for Environmental Decisions, School of BioSciences, University of Melbourne, \\ Melbourne, Victoria 3010 Australia \\ ${ }^{2}$ School of Environmental Sciences, Charles Sturt University, Elizabeth Mitchell Drive, Albury 2640 Australia
}

\begin{abstract}
Biodiversity offsetting schemes permit habitat destruction, provided that losses are compensated by gains elsewhere. While hundreds of offsetting schemes are used around the globe, the optimal timing of habitat creation in such projects is poorly understood. Here, we developed a spatially explicit metapopulation model for a single species subject to a habitat compensation scheme. Managers could compensate for destruction of a patch by creating a new patch either before, at the time of, or after patch loss. Delaying patch creation is intuitively detrimental to species persistence, but allowed managers to invest financial compensation, accrue interest, and create a larger patch at a later date. Using stochastic dynamic programming, we found the optimal timing of patch creation that maximizes the number of patches occupied at the end of a 50-yr habitat compensation scheme when a patch is destroyed after 10 yr. Two case studies were developed for Australian species subject to habitat loss but with very different traits: the endangered growling grass frog (Litoria raniformis) and the critically endangered Mount Lofty Ranges Southern Emu-wren (Spititurus malachurus intermedius). Our results show that adding a patch either before or well after habitat destruction can be optimal, depending on the occupancy state of the metapopulation, the interest rate, the area of the destroyed patch and metapopulation parameters of the focal species. Generally, it was better to delay patch creation when the interest rate was high, when the species had a relatively high colonization rate, when the patch nearest the new patch was occupied, and when the destroyed patch was small. Our framework can be applied to single-species metapopulations subject to habitat loss, and demonstrates that considering the timing of habitat compensation could improve the effectiveness of offsetting schemes.
\end{abstract}

Key words: biodiversity offsets; growling grass frog; habitat compensation; metapopulation; Southern Emu-wren; stochastic dynamic programming.

\section{INTRODUCTION}

Biodiversity offsetting aims to mitigate the environmental impacts of urbanization, agriculture, and mining (ten Kate et al. 2004). Offsetting schemes permit destruction of habitat provided this damage is compensated for elsewhere through activities that aim to achieve "no net loss" of biodiversity (Wissel and Watzold 2010). Although the number of offsetting schemes has significantly increased recently throughout the world, the efficacy of biodiversity offsetting is disputed (Maron 2015). While a theoretically attractive mechanism for achieving both development and conservation objectives, offsetting has been criticized for not encouraging changes in human behavior (Spash 2010), creating perverse incentives (Gordon et al. 2015), and failing to achieve no net loss in practice (Curran et al. 2014).

In habitat compensation schemes, managers generally aim to achieve no net loss of biodiversity by (1) protecting existing habitat from threats, known as averted loss

Manuscript received 4 December 2016; revised 14 September 2017; accepted 23 November 2017. Corresponding Editor: Dianne Brunton.

${ }^{3}$ E-mail: darren_southwell@hotmail.com offsets (Gordon et al. 2011), (2) improving the quality of existing habitat, or (3) creating new habitat elsewhere of equivalent biodiversity value (Maron et al. 2012). In the latter case, the timing of habitat creation relative to its destruction varies. Habitat banking, also referred to as conservation banking, or mitigation banking, requires habitat to be created before it is destroyed (Bekessy et al. 2010). For example, the Wetland Banking scheme in the United States aims to restore or protect large-scale wetlands in order to generate credits that can then be sold to compensate for impacts on similar, nearby wetlands in future (Hough and Robertson 2009). In contrast, some schemes, such as the Melbourne Strategic Assessment in Victoria, Australia (Victorian Government 2012), allow for post-hoc compensation whereby habitat is created at the time of, or after habitat is destroyed elsewhere.

In either habitat banking or post-hoc compensation, the resulting benefits for the ecosystem usually lag behind the creation of habitat (ten Kate et al. 2004, Maron et al. 2012). In the case of post-hoc compensation, habitat creation also often lags behind its destruction, so the required gains significantly lag behind the impacts. Habitat compensation schemes generally aim to minimize this lag to prevent population declines or loss of ecosystem services (Gutrich and Hitzhusen 2004, Johst et al. 2011). 
However, delaying habitat creation may have benefits for species persistence if managers can accrue interest on monetary compensation received for habitat destruction and create more habitat at a later date (assuming the accrued interest is invested directly in habitat creation). Alternatively, managers might be willing to incur interest costs of establishing offsets prior to impacts occurring if the biodiversity benefits are sufficient. To our knowledge, no studies have assessed this trade-off, despite financial interest being a fundamental component of contemporary fiscal systems, and a potentially lucrative means of leveraging compensation funds in offsetting schemes.

Here, we explored the optimal timing of biodiversity offsetting in the context of metapopulation restoration that could be part funded by the accrual of interest on invested compensation monies. We developed case studies for two endangered Australian species with contrasting traits: the growling grass frog (Litoria raniformis) and the Mount Lofty Ranges Southern Emu-wren (Stipiturus malachurus intermedius). Both species persist as metapopulations and are sensitive to habitat loss (Westphal et al. 2003, Heard et al. 2013), but such loss may be offset through habitat creation (new wetlands in the case of the frog; new woodland patches in the case of the Southern Emu-wren). In this study, we (1) developed a framework for modeling metapopulation dynamics for a single-species subject to a biodiversity offsetting scheme, (2) integrated this metapopulation model with a decision-theoretic approach, (3) parameterized our model for $L$. raniformis and $S$. malachurus, and (4) determined the timing of patch creation that maximized the number of patches occupied at the end of the offsetting scheme when financial compensation received for patch loss accrued interest over time.

\section{METHOD}

\section{Formulating the problem}

We assume a standard metapopulation in which habitat patches support demographically discrete populations of a species, but emigration and immigration occurs among populations (Levins 1969, Hanski 1994). Individual populations are prone to extinction, but the resulting vacant patches can be recolonized through time by immigrants from extant populations elsewhere (Levins 1969, Hanski 1994). The annual probability of local population extinction is a function of patch area and quality, while the annual probability of patch colonization is a function of the distance, occupancy status, and size of neighboring patches, termed patch "connectivity."

To incorporate habitat loss and compensation (via offsetting), we assume that a single patch $d$ is destroyed by a developer at time, $t_{d}$. The developer pays the metapopulation manager financial compensation $B_{d, t}$ immediately when patch $d$ is destroyed, with the amount depending on the area of the destroyed patch. We assume managers must compensate for patch loss by adding a new vacant patch to the landscape, which can be created during any year of the offsetting scheme; that is, before, at the time of, or after patch $d$ is destroyed. If managers create a patch before patch loss, they have to borrow the compensation they will receive at time $t_{d}$ at a back-discounted rate and pay interest back on the amount owing. Alternatively, if managers delay creating a new patch, we assume they can invest the financial compensation received at time $t_{d}$ in a conservation trust fund that accrues interest at a fixed rate $\delta$. By delaying action (or initiating it early), managers can create a larger (or smaller) patch, allowing us to ask: "when is it optimal to compensate for patch loss"?

\section{Spatially explicit stochastic patch occupancy model}

We used a generic spatially explicit stochastic patch occupancy model following Day and Possingham (1995). The occupancy state $u$ of the metapopulation at time $t$ was the set of $m$ patches that were occupied at time $t$, given by the $m$-dimensional vector

$$
u(t)=\left[u_{1}(t), u_{2}(t), \ldots, u_{m}(t)\right]
$$

where the elements are binary variables, $u_{i}(t) \in\{0,1\}$. If $u_{i}(t)=1$, then patch $i$ was occupied; if $u_{i}(t)=0$, then patch $i$ was vacant. If all elements of the vector $u_{i}(t)$ equal zero then the metapopulation was extinct.

The probability of transitioning from any occupancy state through extinction was determined by an extinction matrix $\mathbf{X}_{m}$ of dimension $2^{m} \times 2^{m}$. The probability of going from state $u$ to state $v$ in one time step, $x_{u v}$, depended on the probabilities of extinction of individual patches, given by

$$
x_{u v}=\prod_{i=1}^{m} I\left(u_{i}, v_{i}\right)
$$

where the incidence function $I\left(u_{i}, v_{i}\right)$ was the probability that patch $i$ changes from state $u_{i}$ to $v_{i}$ via extinction in the next time step

$$
\begin{aligned}
& I\left(u_{i}, v_{i}\right)= \\
& \begin{cases}1-\varepsilon_{i} & \text { for } u_{i}=1 \text { and } v_{i}=1 \text { (patch } i \text { remains occupied) } \\
\varepsilon_{i} & \text { for } u_{i}=1 \text { and } v_{i}=0 \text { (patch } i \text { becomes empty) } \\
1 & \text { for } u_{i}=0 \text { and } v_{i}=0 \text { (patch } i \text { remains empty) } \\
0 & \text { for } u_{i}=0 \text { and } v_{i}=1 \text { (patch } i \text { cannot become }\end{cases}
\end{aligned}
$$

where $\varepsilon_{i}$ was the patch-specific extinction probability, which depends on landscape-specific variables, such as patch area.

Similarly, we constructed a colonization matrix $\mathbf{R}_{m}$. The probability of going from any population state $u$ to state $v$ by colonization alone, $r_{u v}$ in one time step was given by the product

$$
r_{u v}=\prod_{i=1}^{m} I\left(u_{i}, v_{i}\right)
$$


where the function $I\left(u_{i}, v_{i}\right)$ was the probability that patch $i$ becomes re-colonized in transition from state $u$ to $v$ in the next time step
We found the optimal management policy (i.e., when to add a patch and when to wait) for each occupancy state $u$ and landscape state $z$ of the metapopulation using

$$
I\left(u_{i}, v_{i}\right)= \begin{cases}1-\gamma_{i} & \text { for } u_{i}=0 \text { and } v_{i}=0 \text { (patch } i \text { remains unoccupied) } \\ \gamma_{i} & \text { for } u_{i}=0 \text { and } v_{i}=1 \text { (patch } i \text { becomes occupied) } \\ 1 & \text { for } u_{i}=1 \text { and } v_{i}=1 \text { (patch } i \text { remains occupied) } \\ 0 & \text { for } u_{i}=1 \text { and } v_{i}=0 \text { (patch } i \text { cannot become extinct via colonization) }\end{cases}
$$

where $\gamma_{i}$ was the patch-specific colonization probability, which depends on patch characteristics, such as the configuration, occupancy status, and size of neighboring patches.

The probability of transitioning from any population state $u$ to $v$ was then represented by the product of the colonization matrix $\mathbf{R}_{m}$ and the extinction matrix $\mathbf{X}_{m}$, given by $\mathbf{A}_{m}$. Transitions were modeled as outcomes of Bernoulli trials. We assumed the order of events was colonization followed by extinction.

\section{Patch destruction and creation}

We assumed patch $d$ was destroyed at time $t_{d}$ due to some development project by setting the extinction and colonization probability of the destroyed patch equal to 1 and 0 , respectively, during and after the time of patch loss $\left(t \geq t_{d}\right)$. We assumed the area of the new patch $A_{\text {new }, t}$ was equal to

$$
A_{\text {new }, t}=\frac{A_{d} R}{C}(1+\delta)^{t-t_{d}}
$$

where $A_{d}$ is the area of the destroyed patch, $R$ is the compensation rate per unit area of the destroyed patch, $C$ is the cost of creating habitat per unit area, $\delta$ is the interest rate, $t$ is time, and $t_{d}$ is the time when patch $d$ is destroyed. Here, the financial compensation received at the time of patch loss is equal to $A_{d} R$. We assumed that there was no cost of purchasing land (replicating land donation by the developer or patch creation on public land), that a new patch was initially vacant, and there was no time lag between when a patch is created and when it becomes a functional part of the ecosystem. If $R=C$, a new patch is smaller, equal to, or larger in area than patch $d$ if created before, at the time of, or after habitat loss, respectively. If $R<C$, then a patch added simultaneously with patch loss will be smaller than patch $d$. For simplicity, we assumed $R=C$ and that the interest rate was fixed over time.

\section{Stochastic dynamic programming}

We integrated the generic metapopulation model with stochastic dynamic programming (SDP). SDP is an optimization framework that can be applied where sequential management decisions are made for stochastic systems with a finite number of states (Bellman 1957).
SDP. The landscape state $z$ was defined by all of the possible times a patch could be added to the metapopulation. Therefore, there were $T_{\max }+1$ possible landscape states (because a patch could be added in each year or not at all) and $2^{m}$ possible occupancy states.

The first step of SDP was to define the management objective. We assumed the objective was to maximize the number of patches occupied at the end of the management program, defined by

$$
V_{1}\left((z, t), T_{\max }\right)=n
$$

where $z$ is the landscape state, $T_{\max }$ is the length of the management program and $n$ is the number of occupied patches. A second management objective (maximize the probability that at least one patch is occupied at the end of the offsetting scheme) is presented in Appendix S1. We then determined the best action (i.e., wait or add a patch) for every possible occupancy state $u$ and landscape state $z$ for a metapopulation using the recursive equation

$$
V\left(u, z, t, T_{\max }\right)=\max _{q}\left[\sum_{v=1}^{S} a_{u v}(y) V\left(v, y, t+1, T_{\max }\right)\right]
$$

where $V\left(u, z, t, T_{\max }\right)$ is the value of the metapopulation in state $u$ and landscape state $z$ at time $t, T_{\max }$ is the terminal time, $a_{u v}(y)$ is an element of the transition matrix $\mathbf{A}_{m}(y)$ (the probability of going from population state $u$ to $v$ given that we implement the management strategy $q$ at time $t$ ), and $y$ represents the new landscape state after the strategy $q$ has been implemented. We develop two case studies below to apply our generic metapopulation model integrated with SDP.

\section{Case study 1: growling grass frog}

The endangered growling grass frog (Litoria raniformis) occupies permanent and ephemeral wetlands and slowflowing streams in south-eastern Australia. Population declines have been attributed to several threatening processes, including habitat loss and fragmentation, altered flooding regimes, disease, predation, and drought (Mahony et al. 2013). Around Melbourne, the capital city of Victoria, the most immediate threat facing this species is habitat loss due to urban development, with proposed urban growth to occur in the four regions recognized as remaining strong holds for the species (DEPI 2013). 
To offset these impacts, a habitat retention and creation scheme exists for $L$. raniformis in which developers must retain riparian conservation reserves for the species and pay compensation for destroying habitat outside of these reserves; monies from which will be used to create new wetlands within reserves or improve the quality of existing wetlands therein. The relative effect of mitigation strategies on metapopulation dynamics is relatively well-studied (Heard et al. 2013, Rose et al. 2016); however, the optimal timing of habitat creation if financial compensation received for habitat destruction can accrue interest over time has not been assessed.

Parameterizing the metapopulation model for the growling grass frog.-We estimated the patch-specific extinction and colonization probabilities (described in the section Spatially explicit stochastic patch occupancy model) for L. raniformis from an extensive field survey data set (Heard et al. 2015). We assumed that the probability of extinction was a function of effective wetland area, while the probability of colonization was a function of patch connectivity. This model is a simplification of the model presented by Heard et al. (2013) who assumed extinction also depended on aquatic vegetation cover and patch connectivity. We dropped these two variables from our model so that we could focus on the issue of offset ratios in the context of habitat area, and because they were the weakest predictors of extinction. Applying the logit link, the probability of extinction ( $\varepsilon$ ) for patch $i$ was modeled as

$$
\operatorname{logit}\left(\varepsilon_{i}\right)=\alpha_{\varepsilon}+\beta_{\varepsilon}\left(\mathrm{EA}_{i}\right)
$$

where $\alpha_{\varepsilon}$ is the intercept term, $\beta_{\varepsilon}$ is the coefficient, and EA is the effective area of patch $i$ (a measure of wetland surface area linearly down-weighted by hydroperiod; see Appendix S2).

The probability of colonization $(\gamma)$ for patch $i$ at time $t$ was modeled as

$$
\operatorname{logit}\left(\gamma_{i}\right)=\alpha_{\gamma}+\beta_{\gamma}\left(S_{i, t}\right)
$$

where $S_{i, t}$ is the connectivity of patch $i$ at time $t$. Patch connectivity was calculated using the relationship between inter-patch distance ( $d_{i, j}$, at 10-m increments) and the probability of dispersal for $L$. raniformis, as derived from mark-recapture data by Heard et al. (2012):

$$
w_{i, j}=\left(0.10026 \times d_{i, j}\right)^{-0.719877}
$$

The connectivity of patch $i$ at time $t$ was defined as the distance-weighted sum of the surrounding occupied patches within $1 \mathrm{~km}$ in the previous year

$$
S_{i, t}=\sum w_{i, j} \times o_{j}
$$

where $o_{j}$ is the occupancy status of each neighbor $j$ (one if the patch is occupied, zero otherwise) at time $t-1$. The model was parameterized such that there is a very small chance vacant patches are colonized from outside sources.

Model parameters (mean and 95\% credible intervals) were estimated using data collected at 190 sites across northern Melbourne over $11 \mathrm{yr}$, totaling 2,011 surveys (Table 1). Surveys were conducted to maximize detection rates by carefully considering the timing (i.e., day vs. night) and duration of surveys (i.e., number of repeat visits to a site within a season; Heard et al. 2006). Model fitting was conducted in a hierarchical Bayesian framework to further account for imperfect detection. Further information on data collection, detectability estimates for $L$. raniformis and model fitting can be found in Heard et al. $(2015,2006)$ and Appendix S3, respectively.

\section{Case study 2: Southern Emu-wren}

The critically endangered Southern Emu-wren (Stipiturus malachurus intermedius) forms metapopulations in

TABLE 1. Description and estimate of metapopulation model parameters for the growling grass frog (Litoria raniformis) and Southern Emu-wren (Spititurus malachurus intermedius).

\begin{tabular}{llll}
\hline \hline Parameter & \multicolumn{1}{c}{ Description } & L. raniformis & S. malachurus \\
\hline$m$ & number of patches & 6 & 6 \\
$n$ & number of occupied patches & 3 & 6 \\
$u$ & occupancy state & $2^{m}$ & $2^{m}$ \\
$\mu$ & location parameter & - & $5 \times 10^{5}$ \\
$\rho$ & shape parameter (extinction) & - & $0.6(0.3,0.9)$ \\
$\lambda$ & shape parameter (colonization) & - & $0.0046(0.015,0.0023)$ \\
$\alpha_{\varepsilon}$ & extinction intercept & $1.422(0.197,2.723)$ & - \\
$\beta_{\varepsilon}$ & extinction slope & $-0.541(-0.340,-0.765)$ & - \\
$\alpha_{\gamma}$ & colonization intercept & $-3.718(-3.198,-4.292)$ & - \\
$\beta_{\gamma}$ & colonization slope & $10.26(7.480,13.52)$ & - \\
$\delta$ & interest rate (\%) & $2.5,5,10$ & $2.5,5,10$ \\
$t$ & time step (yr) & 1 & 5 \\
$T_{d}$ & time of habitat destruction (yr) & 10 & 10 \\
$T_{\max }$ & length of time horizon (yr) & 50 & 50 \\
\hline
\end{tabular}

Note: Estimates in brackets represent $95 \%$ credible intervals or values for low and high persistence scenarios. Dashes indicate that a model parameter is not relevant to the species. 
vegetation fragments surrounding swamps in the Mount Lofty Ranges on the Fleurieu Peninsula, South Australia (MLR Southern Emu-wren Recovery Team 1998). Vegetation clearing for agricultural development has destroyed $75 \%$ of the original swamp habitat of this species. As a result, its long-term survival depends on maintaining and re-establishing suitable swamp and heathland vegetation communities.

Although no specific biodiversity offsetting scheme currently exists for this species, South Australian recovery plans list vegetation clearance as a key threat (MLR Southern Emu-wren Recovery Team 1998), and the Australian Federal Government regularly use offsets to manage vegetation clearing (McKenney and Kiesecker 2010). We therefore assumed managers of this species receive financial compensation if existing habitat patches are destroyed, which must be spent creating new patches elsewhere.

Parameterizing the metapopulation model for Southern Emu-wren.-We used a metapopulation model for S. malachurus developed by Westphal et al. (2003). In this model, patch-specific extinction rates were estimated at a 5-yr interval using patch occupancy data. The patch-specific extinction probability over this period was inversely related to patch area

$$
\varepsilon_{i}=\min \left[\frac{0.38}{A_{i}^{\rho}}, 1.0\right]
$$

where $A_{i}$ is the area (ha) for patch $i$ and $\rho$ is a shape parameter influencing the effect of area on extinction probability. The extinction probability is 1 for patches $<0.2$ ha. The probability of patch colonization scales with migration rate $w$. Migration rate is calculated with two assumptions: (1) that the probability of a migrant reaching a given patch $i$ from another $j$ declines exponentially with inter-patch distance and (2) that the number of migrants contributed by patch $j$ scales linearly with its area $\left(A_{j}\right)$. Hence

$$
w_{i}=100 \sum_{m \neq i}^{m} \exp \left(-\lambda d_{i, j}\right) o_{j} A_{j}
$$

where $o_{j}$ equals 1 for occupied patches and 0 for empty patches, $\lambda$ is a shape parameter defining the decay of the dispersal kernel, $d_{i, j}$ is the distance between patches $i$ and $j$, and $m$ is the number of patches in the metapopulation. The probability of colonization is sigmoidally related to the relative number of migrants reaching a patch

$$
\gamma_{i}=\frac{w_{i}^{2}}{w_{i}^{2}+\mu^{2}}
$$

where $\mu$ determines how fast the colonization probability approaches unity with increasing $w_{i}$. Westphal et al. (2003) provide "baseline" estimates of model parameters for this species, as well as alternative values for $\rho$ and $\lambda$ for high and low colonization and extinction scenarios (see Table 1 and Appendix S3). They parameterized the model using maximum likelihood techniques on two years of patch occupancy and individual dispersal data without explicitly accounting for imperfect detection.

\section{Specific metapopulations}

We determined the optimal timing of patch creation for a specific metapopulation for each case study species. For L. raniformis, we selected a metapopulation located in Campbellfield, Melbourne, which consists of a cluster of six patches, including pools along Merri Creek, a water-treatment wetland, a small ephemeral pond, and two large quarries. The configuration and effective area of patches is shown in Fig. 1a. For S. malachurus, we considered the optimal patch relocation strategy for the Cleland Gully metapopulation located in the Mount Lofty Ranges, described by Westphal et al. (2003). This metapopulation is also made up of six patches (Fig. 1b). We set the time step equal to $1 \mathrm{yr}$ for L. raniformis and $5 \mathrm{yr}$ for $S$. malachurus according to the period over which model parameters were estimated.

\section{Development scenarios}

We considered two development scenarios for the Campbellfield and Cleland Gully metapopulations: destruction of either the smallest or largest patch. We assumed either one of these patches was destroyed after $10 \mathrm{yr}\left(t_{d}=10\right)$ of a 50 -yr $\left(T_{\max }\right)$ offsetting scheme (although the influence of alternative choices of $t_{d}$ and $T_{\max }$ are presented in Appendix S4 for S. malachurus). For each development scenario, we positioned the new patch next to the second largest original patch (hereafter referred to as site 1) and assumed it was suitable immediately. However, we also tested the effect of positioning a new patch at the most connected location in the landscape (defined by the centroid of existing patches, hereafter referred to as Site 2; see Appendix S5). These locations were chosen to maximize the chance of the new vacant patch being colonized. We determined the optimal timing of patch creation for three interest rates: $2.5 \%, 5 \%$, and $10 \%$. These rates were selected to lie within the range of term deposit interest rates observed over the last $30 \mathrm{yr}$ in Australia (data from the World Bank, available online; http://data.worldbank.org/indi cator/FR.INR.DPST).

To test the sensitivity of management decisions to parameter uncertainty, we ran the SDP for each species under three metapopulation scenarios: (1) a "baseline" scenario, using our best estimate of colonization and extinction; (2) a "high persistence" scenario, using the highest expected colonization rate combined with the lowest expected extinction rate; and (3) a "low persistence" scenario, using the lowest expected colonization rate combined with the highest expected extinction rate. For L. raniformis, we used the $95 \%$ credible intervals 

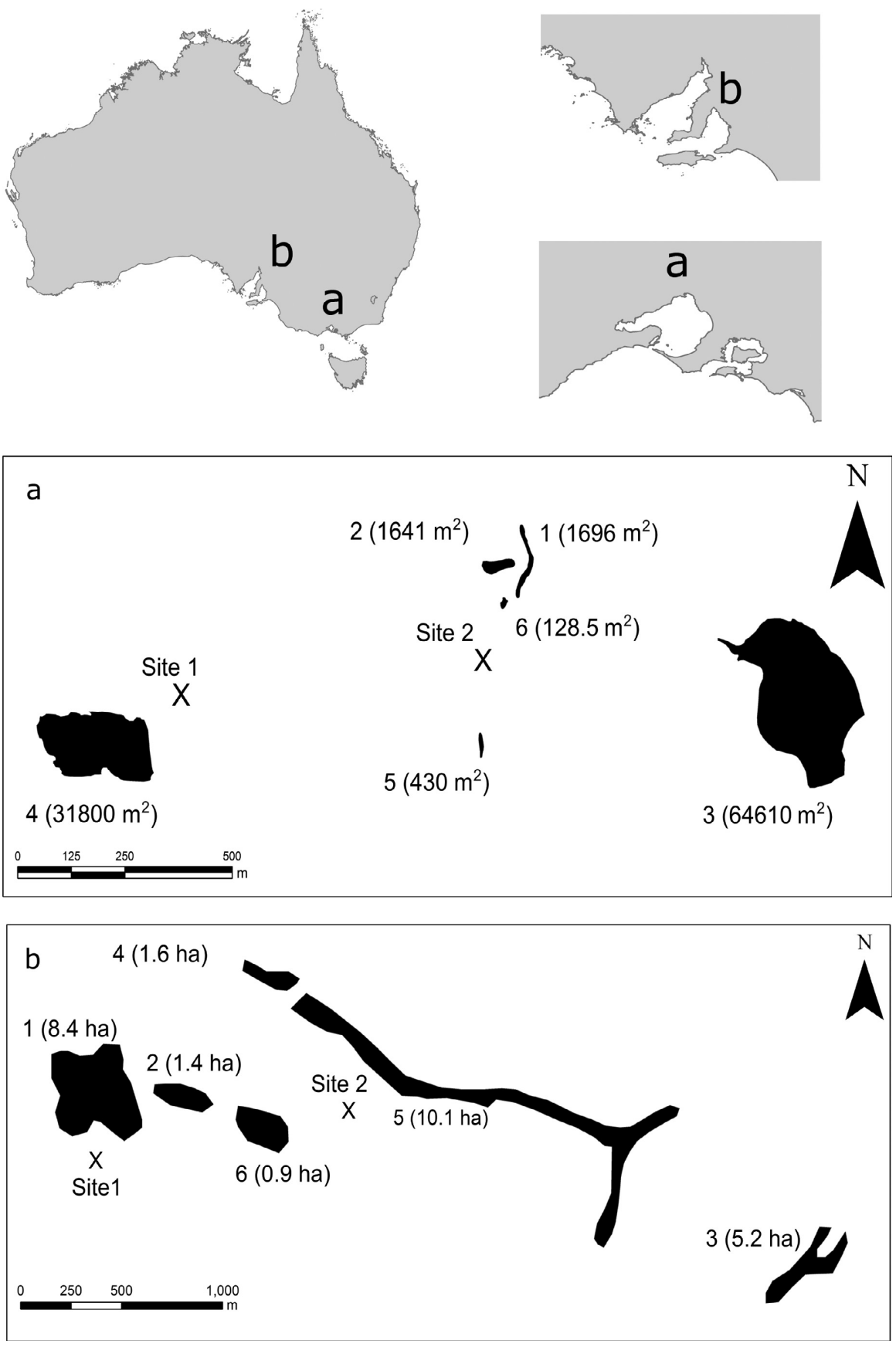

FIG. 1. Configuration of (a) the Campbellfield metapopulation of Litoria raniformis in Melbourne, Australia and (b) the Cleland Gully metapopulation of Spititurus malachurus intermedius in the Mount Lofty Ranges, Australia. Existing patches are shown in black with their area in adjacent text (effective wetland area is shown for L. raniformis). For each metapopulation, either the largest or smallest patch was destroyed after $10 \mathrm{yr}$ of a $50 \mathrm{yr}$ offsetting scheme. New patches were created at two sites indicated with a cross: (1) adjacent to the second largest original patch (site 1), and (2) at the most connected location in each landscape (site 2).

around the extinction and colonization parameters to model low and high persistence scenarios, while for $S$. malachurus, we used alternative values for the shape parameters $\rho$ and $\lambda$ reported by Westphal et al. (2003) (Table 1). All analyses and simulations were conducted using R (R Development Core Team 2014; Data S1).

\section{Simulations}

To illustrate the results of our SDP, we simulated the number of patches occupied in each metapopulation over time for one of the development scenarios described above; that is, when the smallest patch in each 
metapopulation is destroyed and assuming a 5\% interest rate. Simulations were repeated for the baseline, high persistence, and low persistence metapopulation scenarios. For each of these scenarios, we simulated three management alternatives: (1) adding a patch at the time determined by the SDP, (2) always adding a patch immediately when patch $d$ was destroyed (i.e., at $10 \mathrm{yr}$ ), and (3) doing nothing (i.e., not adding a patch to offset loss of patch $d$ ). We ran each simulation 10,000 times with the new patch positioned at site 1 . Initial occupancy states were set to $u=[1,0,1,1,0,0]$ for $L$. raniformis, based on most recent observations of the metapopulation (Heard et al. 2015), and $u=[1,1,1,1,1,1]$ for S. malachurus, as assumed by Westphal et al. (2003).

\section{RESULTS}

Given the large number of occupancy states and management scenarios tested, we present the optimal management policy determined by the SDP for 16 out of the 64 possible occupancy states at each point in time (Figs. 2-4). The occupancy states plotted were chosen to include the initial state of each metapopulation, indicated with a cross. The optimal timing of patch creation for all 64 occupancy states is presented in Appendices S3-S7. The Appendices also include extensions to encompass a second management objective, an alternative location for the new patch, alternative years in which patch loss occurs, and a range of time horizons. In all figures, occupancy states are ordered by the number of occupied patches.

\section{Occupancy state, interest rate and patch area}

The optimal timing of patch creation depended critically on the occupancy state of each metapopulation. For example, it was optimal to create a patch after $39 \mathrm{yr}$ for the $L$. raniformis metapopulation if the smallest patch was destroyed, the interest rate was $5 \%$, the new patch was positioned at site 1 (next to the largest patch), and if the occupancy state remained $u=[1,0,1,1,0,0]$ (Fig. 2c). However, if the metapopulation was initially in a different occupancy state at $t=0$, or transitioned to another occupancy state at some point during the offsetting scheme, the best time to create the patch could change. For instance, if the initial occupancy state of the metapopulation was $u=[1,0,1,1,0,0]$, but the fourth patch becomes extinct, say after $5 \operatorname{yr}(v=[1,0,1,0,0,0])$, then it is optimal to create a patch after $32 \mathrm{yr}$, rather than $39 \mathrm{yr}$ as was the case before the transition occurred (Fig. 2c).

For all of the offsetting scenarios, the number of states in which it was best to delay patch creation increased as the interest rate increased (Figs. 2-4). For example, consider the L. raniformis metapopulation and assume (1) it remained in its last observed occupancy state $(v=[1,0,1,1,0,0])$ and (2) the offsetting scheme entailed adding a new patch at site 1 to compensate for loss of the smallest patch. In this instance, it was optimal to create a wetland with an effective area of $190 \mathrm{~m}^{2}$ after $34 \mathrm{yr}$ if the interest rate was $2.5 \%$ (Fig. 2a). However, it was optimal to wait $41 \mathrm{yr}$ and create a wetland with an effective area of $1,392 \mathrm{~m}^{2}$ if the interest rate was $10 \%$ (Fig. 2c). In the former case, delaying action reduced the probability of extinction of a resident population of the new patch from 0.53 to 0.50 , whereas in the latter case the higher interest rate and longer delay meant the local extinction rate at the new patch reduced to 0.39 .

The area of the destroyed patch influenced the optimal timing of patch creation: it was generally better to delay patch creation when the smallest patch was destroyed compared with the largest patch (Figs. 2, 3). For both species, it was almost always optimal to compensate for loss of the largest patch well before it was destroyed (Fig. 3). The optimal timing of patch creation was sensitive to uncertainty in the metapopulation parameters (Fig. 4). For the L. raniformis metapopulation, it was generally better to delay patch creation for the low persistence scenario, where the colonization rate was low and the extinction rate was high. This is in contrast to the $S$. malachurus metapopulation where there were more states in which it was best to wait under the high persistence scenario.

\section{Management simulations}

Simulations demonstrated a relatively stable trajectory of the Campbellfield L. raniformis metapopulation during a 50-yr offsetting program when we assumed the "baseline" scenario. For this scenario, the population trajectory was similar regardless of when, or if, a patch was created to compensate for loss of a patch (Fig. 5a). On average, 3.199 patches were occupied after $50 \mathrm{yr}$ under the optimal management strategy, 3.189 when a patch was always added after $10 \mathrm{yr}$, and 3.043 under a "do-nothing" scenario. However, the high degree of uncertainty surrounding the metapopulation dynamics for this species resulted in very different trajectories under the low and high persistence scenarios. The low persistence scenario resulted in metapopulation extinction after around $15 \mathrm{yr}$ (Fig. 5c), while almost all of the patches were occupied after $50 \mathrm{yr}$ assuming the high persistence scenario (Fig. 5e).

For S. malachurus, adding a patch at the time determined by the SDP compared with always adding a patch after $10 \mathrm{yr}$ resulted in only a small gain in the number of occupied patches, assuming the baseline scenario (Fig. 5b). However, the benefits of acting optimally compared with sub-optimally were relatively high for the high persistence scenario, when colonization was high and extinction was low. In this case, the number of patches occupied by the $S$. malachurus metapopulation averaged 4.907 under an optimal management strategy, 4.586 when a patch was always added simultaneously with patch destruction, and 3.988 when nothing was done (Fig. 5f). 

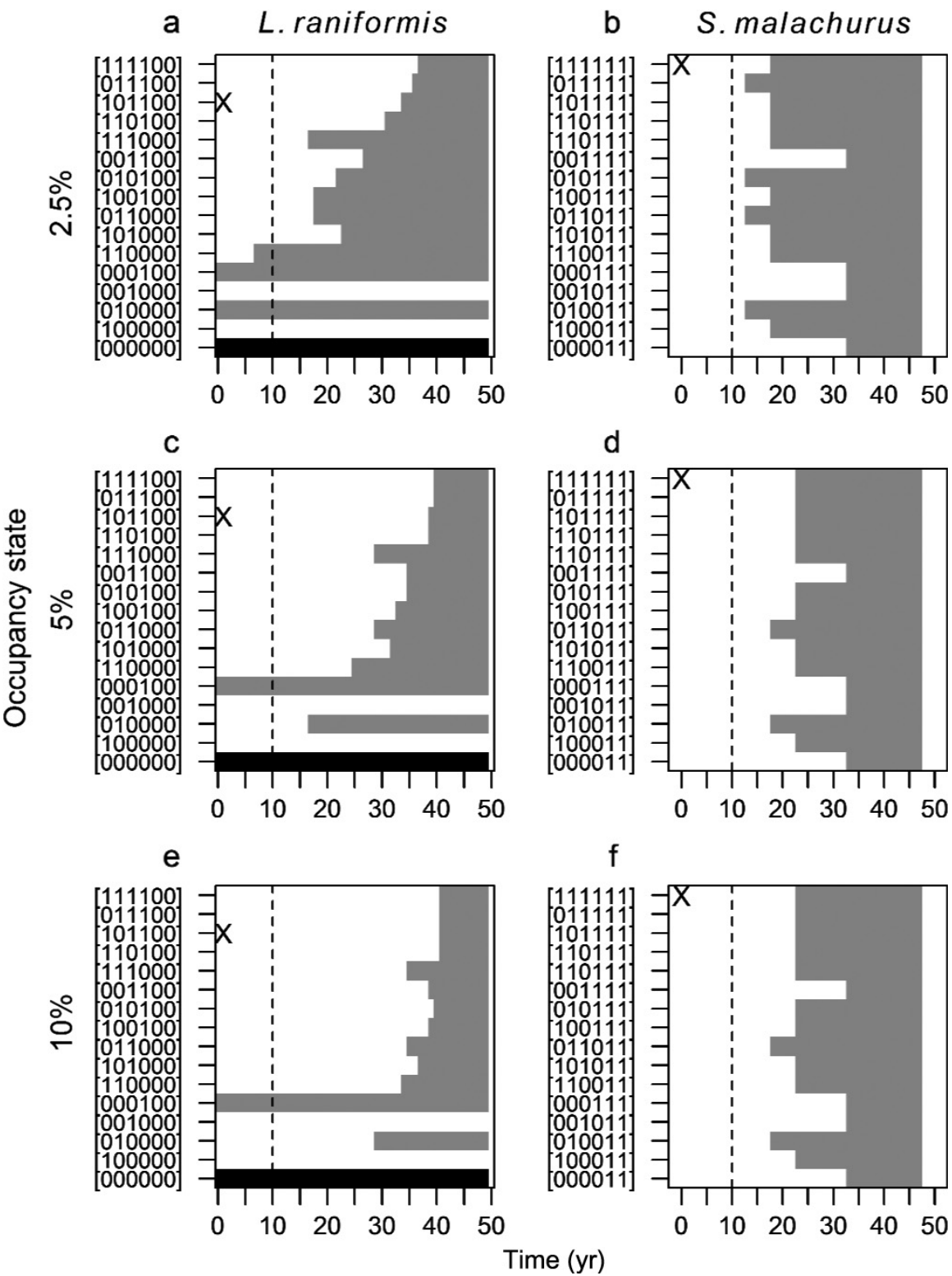

FIG. 2. The optimal management strategy for 16 occupancy states ( $y$-axis) of the Litoria raniformis (left column) and Spititurus malachurus intermedius (right column) metapopulations at each point in time ( $x$-axis) if a patch is yet to be added to the metapopulation. A one in the brackets on the y-axis indicates that patch $\mathrm{i}$ is occupied, a zero indicates it is empty (e.g., [100000] indicates the state where only patch 1 is occupied). The initial occupancy status of each metapopulation is marked with a cross in each panel with the occupancy states ordered by the number of occupied patches. White shading indicates when it is best to wait, gray shading represents when it is best to add a patch. Black shading indicates when the metapopulation is extinct. This scenario assumes the smallest patch is destroyed after $10 \mathrm{yr}$ (indicated by the vertical dashed line) and the new patch is positioned next to the second largest existing patch (site 1). The objective was to maximize the number of patches occupied at the end of a 50 -yr management program. The top, middle, and bottom rows show the optimal management strategy with $2.5 \%, 5 \%$, and $10 \%$ interest rates, respectively.

\section{DISCUSSION}

Biodiversity offsetting aims to create, protect, or restore biodiversity while permitting the destruction of existing habitat to achieve no net loss of biodiversity (ten Kate et al. 2004, Maron et al. 2012). A crucial decision facing managers of these schemes is deciding when to compensate for habitat loss to maximize species persistence. Acting immediately avoids a time lag between patch destruction and creation, which can be detrimental to species persistence (Johst et al. 2011), whereas delaying action might allow managers to invest financial compensation in a trust fund, accrue interest, and create more habitat at a later date. In this study, we assessed this trade-off for two metapopulations threatened by urban expansion and agricultural development. 
a
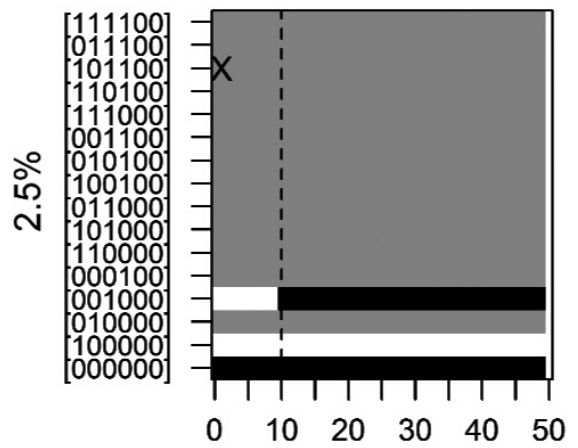

C

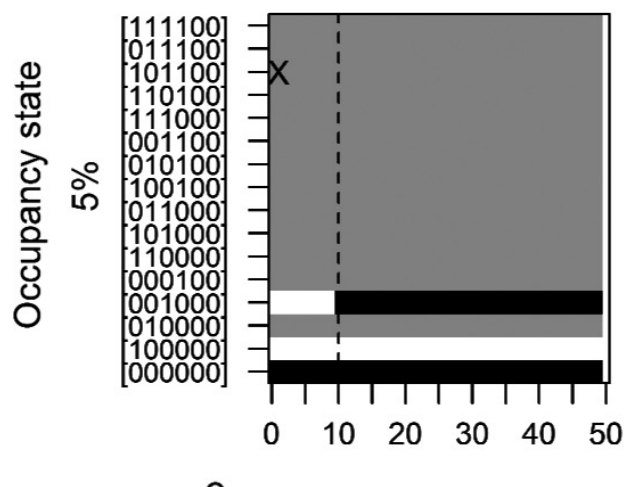

e

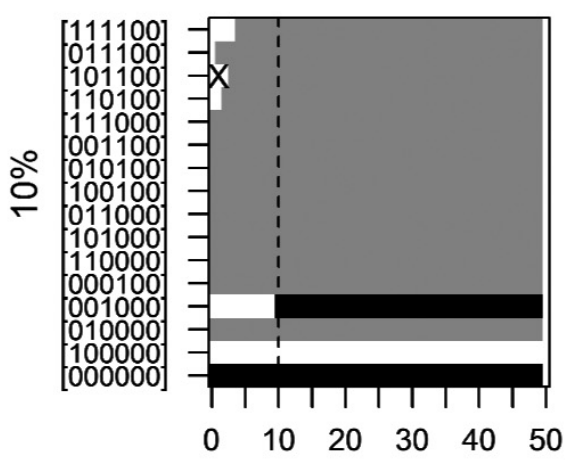

b

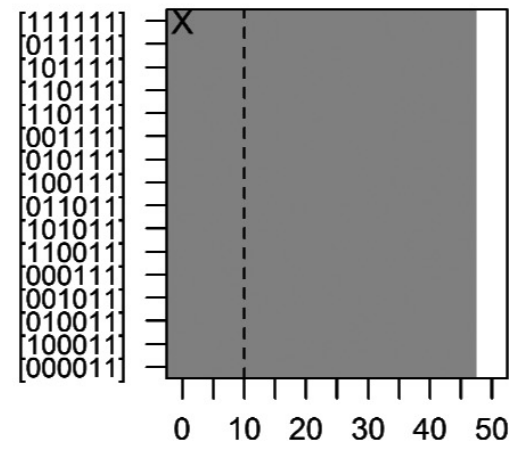

d

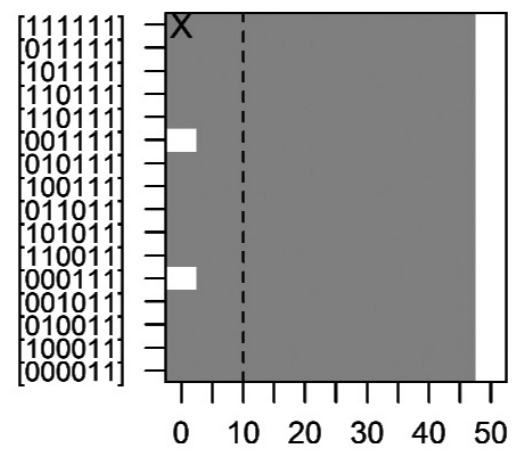

$f$

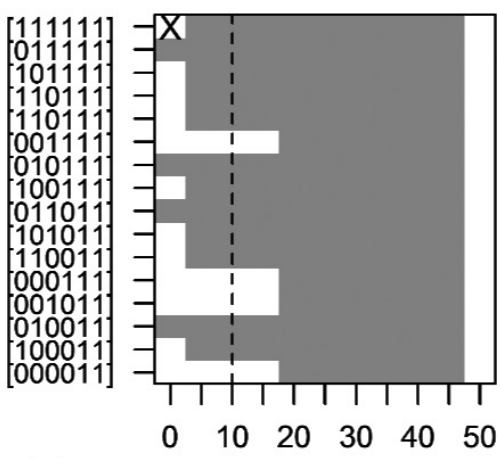

Time (yr)

FIG. 3. The optimal management strategy for 16 occupancy states ( $y$-axis) of the Litoria raniformis (left column) and Spititurus malachurus intermedius (right column) metapopulations at each point in time ( $x$-axis) if a patch is yet to be added to the metapopulation. A one in the brackets on the y-axis indicates that patch i is occupied, a zero indicates it is empty (e.g., [100000] indicates the state where only patch 1 is occupied). The current occupancy status of each metapopulation is marked with a cross in each panel with the occupancy states ordered by the number of occupied patches. White shading indicates when it is best to wait, gray shading represents when it is best to add a patch. Black shading indicates when the metapopulation is extinct. This scenario assumes the largest patch is destroyed after $10 \mathrm{yr}$ (indicated by the vertical dashed line) and the new patch is positioned next to the largest remaining patch (site 1). The objective was to maximize the number of patches occupied at the end of a 50-yr management program. The top, middle, and bottom rows show the optimal management strategy with $2.5 \%, 5 \%$, and $10 \%$ interest rates, respectively.

We showed that it can be optimal to either create a patch prior to habitat loss, or delay patch creation in order to accrue interest, depending on the species of interest, the occupancy state of the metapopulation, and the management scenario. More specifically, the optimal timing of patch creation depended on the species traits, uncertainty around the extinction and colonization rates, the interest rate, the amount of habitat destroyed, and the management objective.

The optimal timing of patch creation depended critically on the occupancy state of each metapopulation. It was generally better to delay action if the patch nearest the site of the new patch was occupied, or conversely, add a patch immediately if the nearest patch was vacant (see 


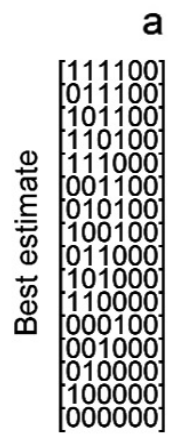

a

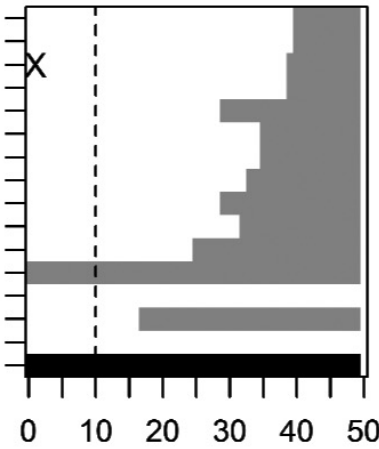

C
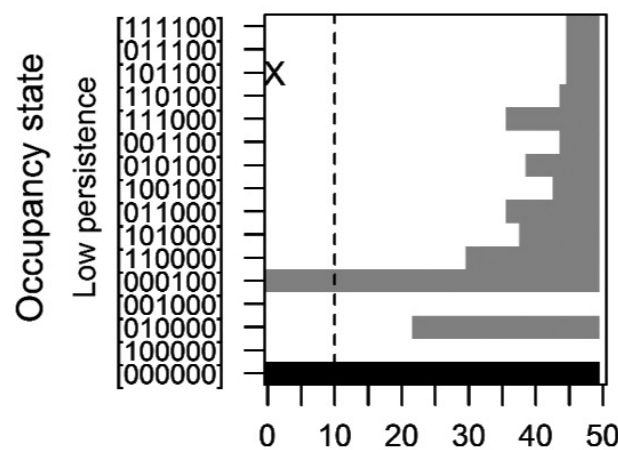

e

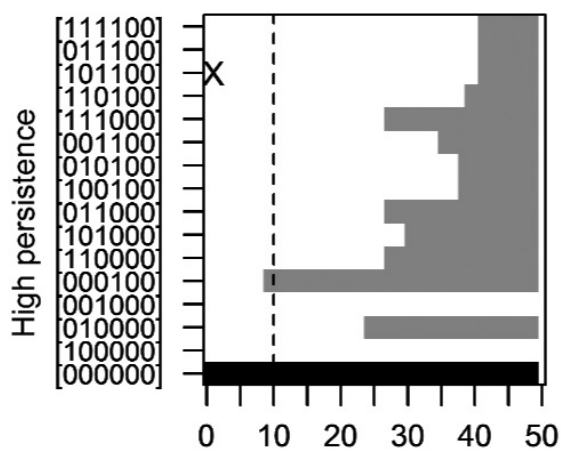

b

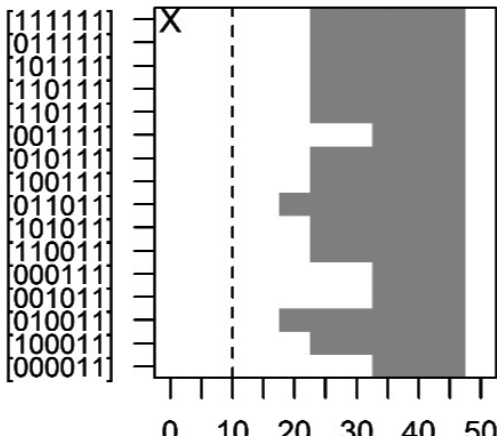

d

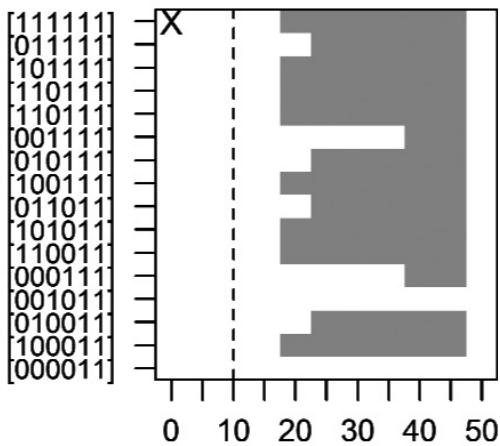

$f$
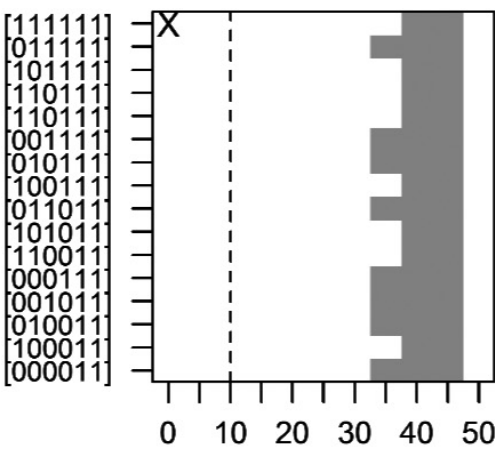

Time (yr)

FIG. 4. The optimal management strategy for 16 occupancy states ( $y$-axis) of the Litoria raniformis (left column) and Spititurus malachurus intermedius (right column) metapopulations at each point in time ( $x$-axis) if a patch is yet to be added to the metapopulation. A one in the brackets on the y-axis indicates that patch i is occupied, a zero indicates it is empty (e.g., [100000] indicates the state where only patch 1 is occupied). The current occupancy status of each metapopulation is marked with a cross in each panel with the occupancy states ordered by the number of occupied patches. White shading indicates when it is best to wait, gray shading represents when it is best to add a patch. Black shading indicates when the metapopulation is extinct. This scenario assumes the smallest patch is destroyed after $10 \mathrm{yr}$ (indicated by the vertical dashed line), the new patch is positioned at site 1, and a $5 \%$ interest rate. The objective was to maximize the number of patches occupied at the end of a 50-yr management program. The top row represents our best estimate of model parameters (baseline scenario), the middle row represents a low persistence scenario, while the bottom row represents a high persistence scenario.

Appendix S6). This result stems from the fact that a patch added next to a vacant patch required more time to be colonized than when next to an occupied one. However, the degree to which this was the case was influenced by the colonization rate, the extinction rate, and the number of occupied patches. For instance, the L. raniformis metapopulation was more sensitive to occupancy status than the S. malachurus metapopulation because the dispersal kernel for this species decays steeply with increasing distance. This meant a new patch had very little chance of being colonized if it was further than $\sim 200 \mathrm{~m}$ from occupied neighbors. In contrast, a patch added to 
a

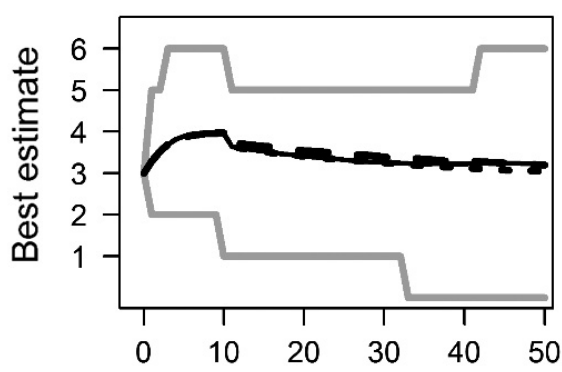

C

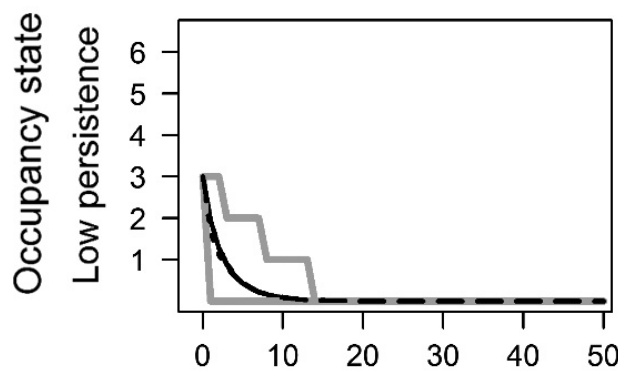

e

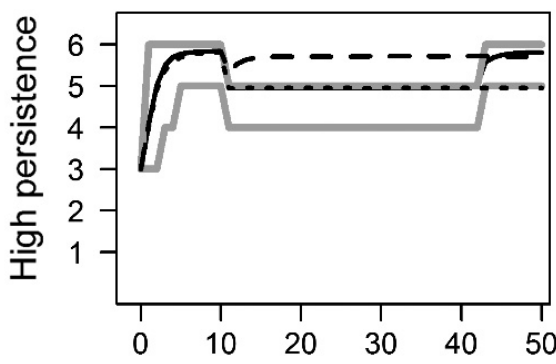

b S. malachurus

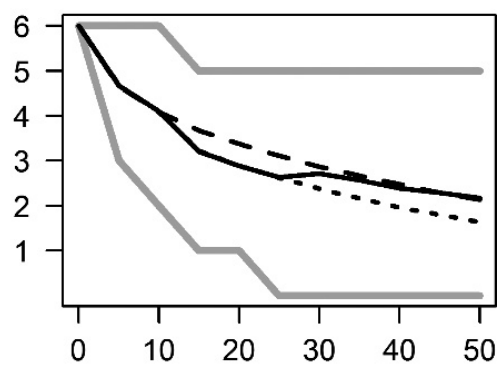

d

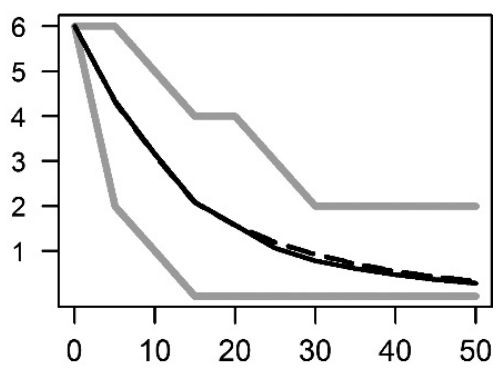

f

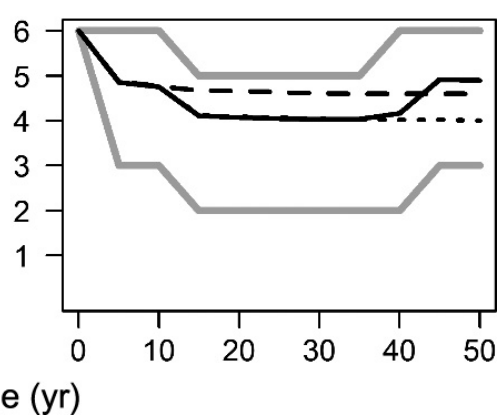

FIG. 5. The mean number of occupied patches when managing the Litoria raniformis (left column) and Spititurus malachurus intermedius (right column) metapopulations over a 50-yr management program from 10,000 simulations. Simulations assumed the smallest patch was destroyed in year 10, financial compensation accrued interest at 5\% per annum, the new patch was positioned next to the second largest existing patch (site 1), with an initial occupancy state $u=[1,0,1,1,0,0]$ for L. raniformis and $u=[1,1,1,1,1,1]$ for $S$. malachurus intermedius. The top row represents our best estimate of model parameters (baseline scenario), the middle row represents the low persistence scenario, while the bottom row represents the high persistence scenario. Solid lines represent the optimal management strategy derived using stochastic dynamic programming, dashed black lines a sub-optimal strategy (patch always added after $10 \mathrm{yr}$ ), and dotted lines doing nothing (i.e., not adding a patch). Gray lines represent the $2.5 \%$ and $97.5 \%$ quantiles around the optimal management strategy.

the $S$. malachurus metapopulation had a higher chance of being colonized by more distant patches, making management decisions less sensitive to the occupancy state, particularly that of the closest patch.

The sensitivity of management decisions to the occupancy state of the metapopulation is important because it means that managers must know which patches are occupied with certainty during each time step until a new patch is created. In practice, correctly knowing the occupancy status of each patch may be difficult for cryptic or secretive species that have detection probabilities much less than one (Guillera-Arroita and Lahoz-Monfort 2012). If detection rates for a species are known, carefully considering the timing, intensity, and duration of surveys can reduce the chance of false negatives and maximize the chance of creating a patch at the optimal time. For example, detectability of $L$. raniformis is relatively well understood: detection probability exceeds 0.9 when nocturnal surveys are repeated over two or three nights (Heard et al. 2006). However, detectability rates will not always be known (as was the case for $S$. malachurus), making it unclear how much monitoring effort is required to detect a species that is present in a patch. Failing to account for detectability will likely bias parameter estimates and will likely increase the chance of incorrectly identifying the true occupancy state, which 
could result in creating habitat at a sub-optimal time. Monitoring the occupancy status of patches during each time step will add additional costs to an offsetting scheme not considered in this study. Further work could expand our framework to explore the trade-off between spending compensation funds on monitoring the occupancy status of existing patches and creating a new patch to offset destruction of another.

For both species, it was almost always optimal to compensate for loss of the largest patch well before it was destroyed. This result is not surprising: the benefit of large patches is well recognized in the metapopulation literature (Verboom et al. 2001, Caruso et al. 2010). Large patches support larger populations, play a greater role in network stability, and are less sensitive to environmental stochasticity. In particular, the largest patches in these two case studies support very resilient local populations and provide a source of colonists to the smaller "satellite" patches that make up the networks. However, the importance of large patches and the ability of metapopulations to tolerate their temporary loss will also depend on the number of patches, the configuration of patches (Pilgrim et al. 2013), and the position of the destroyed and new patch relative to existing patches. For example, a large patch may have little influence on metapopulation persistence if it is located well away from other patches. In our study, it was almost always optimal to offset loss of the largest patch well before it was destroyed. In practice, compensating for patch destruction before it occurs will require managers to have prior knowledge about an offsetting scheme (i.e., the location and timing of future patch loss). They then must borrow the financial compensation at a back-discounted rate, or receive an early discounted rate from the developers, and pay back the amount owing in interest. While anticipating future development might require considerable foresight by managers, our results show that actively compensating for habitat loss before it occurs rather than after can improve metapopulation persistence, particularly if the destruction of large patches is unavoidable.

Our simulations demonstrated that the benefits of acting optimally compared with always adding a patch simultaneously with patch loss, or doing nothing, was highly sensitive to the metapopulation dynamics. There was little benefit in considering the optimal timing of patch creation for the baseline and low persistence scenarios. This is because the potential benefits of delaying action were rarely realized; that is, the new patch had little chance of being colonized regardless of its area. In contrast, adding a patch at the time determined by the SDP improved the number of occupied patches for the high persistence scenarios, when colonization was high and extinction was low. This was especially the case for the S. malachurus metapopulation. Adding a patch at a time determined by the SDP resulted in a $6.99 \%$ increase in the number of occupied patches after $50 \mathrm{yr}$. However, the gains in acting optimally compared with sub-optimally was highly sensitive to the time horizon and the year when the patch is destroyed. For example, we ran additional simulations for S. malachurus (under the high persistence scenario) by varying the time horizon (20-100 yr) and year in which patch loss occurred (5-45 yr) and found that the benefits of acting optimally increased as the time between patch loss and the end of the offsetting scheme increased (Appendix S4). Extending the time horizon from 50 to $100 \mathrm{yr}$ increased the benefits of acting optimally compared with sub-optimally from $6.99 \%$ to $10.54 \%$.

Delaying patch creation may have benefits beyond financial interest on compensation funds. Waiting may provide managers with the opportunity to resolve key uncertainties in metapopulation dynamics or restoration strategies that influence management decisions. For example, managers could delay management actions while compensation funds accrue interest but still learn about the colonization and extinction dynamics of a metapopulation by observing the turn-over rate of patches (Southwell et al. 2016). This would allow managers to update their estimates of the colonization and extinction parameters in the metapopulation model component of our framework, which would lead to more effective allocation of invested compensation funds. Delaying action might also allow managers to refine habitat restoration techniques based on the outcomes of ongoing efforts elsewhere on similar or related species. For example, managers of $L$. raniformis continue to learn about how best to create wetlands for this species by monitoring existing habitat or newly created wetlands that are naturally colonized by the frog, and by learning from research and management programs for related species (Heard et al. 2010, 2013, 2015, DEPI 2013). It is critical, however, that the value of delaying action is weighed against the inflated risk of extinction from delaying habitat compensation, and hence the risk of acting too late.

If delaying action, we assumed managers deposit compensation funds received for habitat loss into a trust fund with a fixed interest rate agreed upon with a financial institution. Modeling time-varying interest rates or different forms of time-discounting is technically possible within our framework (Moore et al. 2008, Laitila et al. 2014); however, predicting the direction and magnitude of variation in interest rates over time horizons relevant to ecological systems is notoriously difficult, if not impossible. We chose to model a fixed interest rate to represent the expected long-running average in this variation over time, and selected three plausible rates to test the sensitivity of management decisions to this parameter. Our choice to model a fixed interest rate for the life of the investment period is also consistent with the investment approach that managers will have available to them to increase monetary compensation. In addition, we assumed that the lending rate (relevant if a new patch is created before patch $d$ is destroyed) was equal to the deposit rate (relevant if patch creation is delayed and compensation funds are invested). Our framework can easily be extended to account for differences in these rates. For example, we present scenarios in 
Appendix S7 where the deposit rate is less than the lending rate and show that these differences had little influence on the optimal timing of management decisions. Expanding the economic component of our framework to incorporate more complicated market mechanisms constitutes an important area of further research, although conservation trust funds are often highly individualized devices.

We made a number of further assumptions in this study. First, we assumed certain benefits from new patches; that they provided "like-for-like" habitat functionality. In practice, other factors might mean new patches are never as suitable as existing habitat. This is a crucial consideration for offsetting schemes in general, and must be carefully considered when designing real world schemes (Hobbs et al. 2011). Second, we ignored a time lag that may occur between when habitat is created and when it plays a functional role for the focal species (Vesk et al. 2008). Accounting for time lags will likely require managers to create habitat sooner than what was reported here, so that a new patch has sufficient time to be colonized before the end of the offsetting scheme. Time lags will likely be less relevant to $L$. raniformis than S. malachurus because of the habitat being created (wetlands vs. terrestrial vegetation communities). Litoria raniformis is known to flourish in some newly created wetlands, when vegetation diversity is often high due to initial planting, when solar insolation is high, when stormwater pollutants are minimal, and when predatory fish are absent (Heard et al. 2010). Third, we did not consider the option to restore the quality of existing patches (averted offset loss), the cost of purchasing land or the administration costs of an offsetting fund. Fourth, we did not explicitly consider an increase in the cost of patch creation through time. Instead, we assumed that the interest rate acting on the compensation fund was relative to any trend in the cost of creating habitat. Fifth, although new patches were positioned favorably, our work could be extended to explore the effect of habitat creation at more strategic positions in the network or by dividing the fund to create more than one new patch at multiple locations.

Finally, we set the compensation rate $R$ equal to the cost of creating habitat $C$, so that the equivalence ratio in terms of habitat area was 1:1 if a patch was added simultaneously with patch loss. By doing this, we assumed that no net loss was achieved in terms of habitat area because the new patch was equal in area to the destroyed patch. Many authors have shown that offset ratios larger than 1:1 are likely required to ensure sufficient biodiversity value in future (Moilanen et al. 2009). For example, Pickett et al. (2013) found that a 19:1 equivalence ratio was required to offset habitat loss for the green and golden bell frog (Litoria aurea), the sister species of $L$. raniformis. We note, however, that their ratio did not account for the spatial arrangement of new wetlands relative to existing habitat. Wetlands spaced sparsely, or ad hoc with relation to existing patches, will require a higher offset ratio because they will have a lower chance of being colonized and contribute fewer successful migrants to the network. Our approach specifically considers the metapopulation dynamics of the focal species, and therefore provides explicit information about offsetting requirements, rather than relying on offset ratios that make implicit assumptions about population or metapopulation viability.

\section{Conclusion}

Hundreds of biodiversity offset schemes are used around the world with little consideration of the optimal timing of habitat creation relative to its destruction. We show that when maximizing the number of patches occupied at the end of an offsetting scheme, the optimal timing of habitat compensation can depend on a species' traits, the configuration of habitat, area of the destroyed patch, the occupancy state, and the interest rate. Considering the optimal timing of habitat compensation will likely benefit highly mobile species that can readily colonize newly created habitat. Knowing when to compensate for patch loss a priori is difficult given the number of factors that can influence the optimal timing of patch creation. This paper provides a framework that can be applied to any metapopulation subject to habitat compensation schemes to determine if delaying action is worthwhile, allowing the optimal timing of these schemes to be explicitly assessed when planning for metapopulation persistence.

\section{AcKnowledgments}

This work was funded by the Australian Research Council Centre of Excellence for Environmental Decisions (CEED). G. W. Heard received support from the Victorian Postdoctoral Research Fellowship from the Victorian Department of Economic Development, Jobs, Transport and Resources. We thank Ascelin Gordon and four anonymous reviewers for providing useful comments on the manuscript.

\section{Literature Cited}

Bekessy, S. A., B. A. Wintle, D. B. Lindenmayer, M. A. McCarthy, M. Colyvan, M. A. Burgman, and H. P. Possingham. 2010. The biodiversity bank cannot be a lending bank. Conservation Letters 3:151-158.

Bellman, R. 1957. Dynamic programming. Princeton University Press, Princeton, New Jersey, USA.

Caruso, A., G. Thor, and T. Snall. 2010. Colonization-extinction dynamics of epixylic lichens along a decay gradient in a dynamic landscape. Oikos 119:1947-1953.

Curran, M., S. Hellweg, and J. Beck. 2014. Is there any empirical support for biodiversity offset policy? Ecological Applications 24:617-632.

Day, J. R., and H. P. Possingham. 1995. A stochastic metapopulation model with variability in patch size and position. Theoretical Population Biology 48:333-360.

DEPI. 2013. Sub-regional species strategy for the growling grass frog. DEPI, Melbourne, Victoria, Australia.

Gordon, A., J. W. Bull, C. Wilcox, and M. Maron. 2015. Perverse incentives risk undermining biodiversity offset policies. Journal of Applied Ecology 52:532-537. 
Gordon, A., W. T. Langford, J. A. Todd, M. D. White, D. W. Mullerworth, and S. A. Bekessy. 2011. Assessing the impacts of biodiversity offset policies. Environmental Modelling \& Software 26:1481-1488.

Guillera-Arroita, G., and J. J. Lahoz-Monfort. 2012. Designing studies to detect differences in species occupancy: power analysis under imperfect detection. Methods in Ecology and Evolution 3:860-869.

Gutrich, J. J., and F. J. Hitzhusen. 2004. Assessing the substitutability of mitigation wetlands for natural sites: estimating restoration lag costs of wetland mitigation. Ecological Economics 48:409-424.

Hanski, I. 1994. A practical model of metapopulation dynamics. Journal of Animal Ecology 63:151-162.

Heard, G. W., M. A. McCarthy, M. P. Scroggie, J. B. Baumgartner, and K. M. Parris. 2013. A Bayesian model of metapopulation viability, with application to an endangered amphibian. Diversity and Distributions 19:555-566.

Heard, G. W., P. Robertson, and M. P. Scroggie. 2006. Assessing detection probabilities for the endangered growling grass frog (Litoria raniformis) in southern Victoria. Wildlife Research 33:557-564.

Heard, G. W., M. P. Scroggie, and N. Clemann. 2010. Guidelines for managing the endangered Growling Grass Frog in urbanising landscapes. Arthur Rylah Institute for Environmental Research, Heidelberg, Germany.

Heard, G. W., M. P. Scroggie, and B. S. Malone. 2012. The life history and decline of the threatened Australian frog, Litoria raniformis. Austral Ecology 37:276-284.

Heard, G. W., C. D. Thomas, J. A. Hodgson, M. P. Scroggie, D. S. L. Ramsey, and N. Clemann. 2015. Refugia and connectivity sustain amphibian metapopulations afflicted by disease. Ecology Letters 18:853-863.

Hobbs, R. J., L. M. Hallett, P. R. Ehrlich, and H. A. Mooney. 2011. Intervention ecology: applying ecological science in the twenty-first century. BioScience 61:442-450.

Hough, P., and M. Robertson. 2009. Mitigation under Section 404 of the Clean Water Act: Where it comes from, what it means. Wetlands Ecology and Management 17:15-33.

Johst, K., M. Drechsler, A. J. A. van Teeffelen, F. Hartig, C. C. Vos, S. Wissel, F. Watzold, and P. Opdam. 2011. Biodiversity conservation in dynamic landscapes: trade-offs between number, connectivity and turnover of habitat patches. Journal of Applied Ecology 48:1227-1235.

Laitila, J., A. Moilanen, and F. M. Pouzols. 2014. A method for calculating minimum biodiversity offset multipliers accounting for time discounting, additionality and permanence. Methods in Ecology and Evolution 5:1247-1254.

Levins, R. 1969. Some demographic and genetic consequences of environmental heterogeneity for biological control. Bulletin of the Entomological Society of America 15:237-240.

Mahony, M. J., et al. 2013. Identifying conservation and research priorities in the face of uncertainty: a review of the threatened bell frog complex in eastern Australia. Herpetological Conservation and Biology 8:519-538.

Maron, M. 2015. Stop misuse of biodiversity offsets. Nature 523:401-403.

Maron, M., R. J. Hobbs, A. Moilanen, J. W. Matthews, K. Christie, T. A. Gardner, D. A. Keith, D. B. Lindenmayer, and C. A. McAlpine. 2012. Faustian bargains? Restoration realities in the context of biodiversity offset policies. Biological Conservation 155:141-148.

McKenney, B. A., and J. M. Kiesecker. 2010. Policy development for biodiversity offsets: a review of offset frameworks. Environmental Management 45:165-176.

MLR Southern Emu-wren Recovery Team. 1998. Recovery Plan for the Mount Lofty Ranges Southern Emu-wren Stipiturus malachurus intermedius: 1999-2003. Report to the Regional Wildlife Programs Section, Wildlife Australia. Conservation Council of South Australia, Adelaide, South Australia, Australia.

Moilanen, A., A. J. A. van Teeffelen, Y. Ben-Haim, and S. Ferrier. 2009. How much compensation is enough? A framework for incorporating uncertainty and time discounting when calculating offset ratios for impacted habitat. Restoration Ecology 17:470-478.

Moore, A. L., C. E. Hauser, and M. A. McCarthy. 2008. How we value the future affects our desire to learn. Ecological Applications 18:1061-1069.

Pickett, E. J., M. P. Stockwell, D. S. Bower, J. I. Garnham, C. J. Pollard, J. Clulow, and M. J. Mahony. 2013. Achieving no net loss in habitat offset of a threatened frog required high offset ratio and intensive monitoring. Biological Conservation 157:156-162.

Pilgrim, J. D., et al. 2013. A process for assessing the offsetability of biodiversity impacts. Conservation Letters 6:376-384.

R Development Core Team. 2014. R: a language and environment for statistical computing. R Foundation for Statistical Computing, Vienna, Austria.

Rose, L. E., G. W. Heard, Y. E. Chee, and B. A. Wintle. 2016. Cost-effective conservation of an endangered frog under uncertainty. Conservation Biology 30:350-361.

Southwell, D. M., C. E. Hauser, and M. A. McCarthy. 2016. Learning about colonization when managing metapopulations under an adaptive management framework. Ecological Applications 26:279-294.

Spash, C. L. 2010. The brave new world of carbon trading. New Political Economy 15:169-195.

ten Kate, K., J. Bishop, and R. Bayon. 2004. Biodiversity offsets: views, experiences and the business case. IUCN, Gland, Switzerland and Cambridge, UK and Insightful Investment, London, UK.

Verboom, J., R. Foppen, P. Chardon, P. Opdam, and P. Luttikhuizen. 2001. Introducing the key patch approach for habitat networks with persistent populations: an example for marshland birds. Biological Conservation 100:89-101.

Vesk, P. A., R. Nolan, J. R. Thomson, J. W. Dorrough, and R. Mac Nally. 2008. Time lags in provision of habitat resources through revegetation. Biological Conservation 141:174-186.

Victorian Government. 2012. Victoria's native vegetation management: a framework for action. Victorian Government, Melbourne, Victoria, Australia.

Westphal, M. I., M. Pickett, W. M. Getz, and H. P. Possingham. 2003. The use of stochastic dynamic programming in optimal landscape reconstruction for metapopulations. Ecological Applications 13:543-555.

Wissel, S., and F. Watzold. 2010. A conceptual analysis of the application of tradable permits to biodiversity conservation. Conservation Biology 24:404-411.

SUPPORTING INFORMATION

Additional supporting information may be found online at: http://onlinelibrary.wiley.com/doi/10.1002/eap.1666/full 